\title{
EVENT DATA VISUALIZATION THROUGH PROCESS MINING:A CASE FOR EMERGENCY MEDICAL SERVICE SYSTEM IN ADANA
}

\begin{abstract}
Nuşin $U N C U^{1}$
Increasing amount of data enables researchers the opportunity of applying new scientific methods to manage and visualize the systems and processes. Process mining is an emerging tool for discovering real processes using event data of complex systems such as communication, information, health care systems, transportation and etc. Emergency Medical Service (EMS) system is an integral part of health care systems and aims to respond cases on time in order to decrease mortality. Although the EMS system process is assumed to be known, related data may indicate some deviations from the real process. Aim of this study is to discover and visualize EMS system in Adana city, in Turkey. EMS system event logs are filtered and visualized by using plug-ins in ProM platform such as Simple Heuristic Filtering plug-in and Log visualizer, respectively. Other plug-ins such as Fuzzy Miner and Inductive Miner are used for discovering process model. The deviations are obtained in EMS system process model showing irregular or rare events that cannot be representable throughout the process. The results indicate that the process of transportation between hospitals should be investigated in order to improve the process of Adana EMS system.
\end{abstract}

Key words: Process mining, Petri nets, Fuzzy miner, Inductive miner, Event $\log s$

\section{Introduction}

Emergency Medical Service (EMS) system is an integral part of health care systems. EMS system can be classified as Anglo-American "Scoop and Run" and Franco-German "Stay and Play". While the first one is a paramedic-based system, the latter is physician based system. The key difference between these two systems is that the health staff and equipment are brought to the case in Franco-German systems, while the case is brought to the health center in Anglo-American systems. A more detailed discussion of these two systems can be found in [1]. In Turkey, EMS system works based on FrancoGerman discipline. For all the types of EMS system, to respond at least required percentage of cases in a predefined time interval is the key performance criterion in order to decrease the mortality.

The process of EMS system starts with receiving an emergency call to a call center where there is a standard call evaluation process to dispatch the available emergency unit to the location within an acceptable time. The nearest and available ambulance is dispatched to the scene including paramedics who perform the first aid. Then the patient is transported to the nearest appropriate hospital and the

1 Department of Industrial Engineering, Adana Alparslan Türkeş Science and Technology University, Adana, Turkey, (nuncu@atu.edu.tr) Dhttps://orcid.org/0000-0003-3030-3363 
process ends after the ambulance turns back to its destination station. The entire process of EMS systems is illustrated in Figure 1.

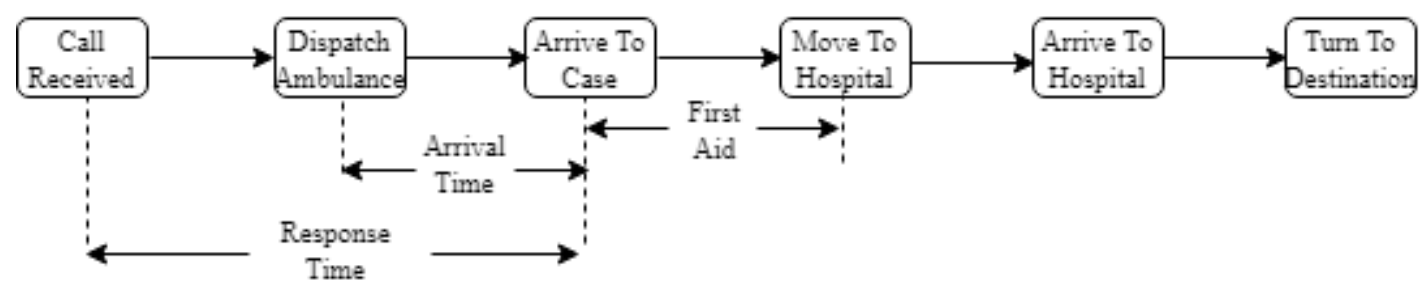

Figure 1. EMS system process chart

Although the EMS system process is assumed to be known, the data may indicate some deviations from the real process. The real process can be discovered from this data by process mining techniques due that the process may be the one that is not what exactly known. Increasing amount of data enables researchers to reach the unknown parts of processes in complex real systems. The idea behind the process mining is to capture real processes and actions from event data. The approach is not just datacentric but also event data-centric. The main steps of process mining are discovering model, conformance checking and enhancement [2]. Discovering model means to explore process model from event logs. However, not all the recorded data are always ready for discovering model phase and data preprocessing in process mining is a time consuming issue. This phase enables to get more accurate data and transfer data in a compatible format while applying process mining techniques.

Main phase in process mining is discovering process model from data. Alpha algorithm is one of the model discovery tools used to explore the causality from a set of event logs [3]. However, alpha algorithm has some limitations while dealing with short loops. In order to tackle with this problem several extensions of alpha algorithm was improved as well other process discovery algorithms such as heuristic miner [4], genetic process mining [5], region based mining [6], inductive miner [7] and interactive process miner [8]. Inductive mining algorithm is one of the Divide-and-Conquer approach aims to split the event log that is difficult to be decomposed by other mining methods. Inductive mining is one of the leading approaches due to its flexibility, formal guarantees and scalability [2].

Process mining tools have been used recently to discover process models from real event data. Process models are useful representations of systems. Transition systems, Petri Nets, WF-Nets (Workflow Nets), BPMN (Business Process Modelling Notation), C-Nets (Causal Nets), EPCs (Eventdriven Process Chains ), YAWL (Yet Another Workflow Language) and process trees are different ways of generating process models. Among these, Petri Nets are simple and intuitive graphical notation to be executable and applicable to many systems [2]. The early application of Petri Nets in EMS system is found in Xiong et al. [9]. Petri Nets are useful for modelling nondeterministic and complex systems. The main problem handled by researchers is to increase the performance of the system after investigating the actual situation based on the effectiveness and resource utilization.

The applications of process mining tools and methodologies in health care systems are deeply explained in Mans et al. [10]. The survey on case studies can be found in Rojas et al. [11]. A number of related applications of mining techniques are exist in [12-16].

The main idea that emerges in the context of process mining is to investigate a general process model that can represent all possible flows with reasonable level of fitness, precision, generalization and simplicity. An overview of challenges of process mining can be found in [17]. 
In this study, although the EMS process flow is almost known, deviations from the model and the mostly or rarely occurring traces are aimed to be investigated. The study is constructed in four section. After an introduction section with a brief literature review, EMS system in Adana is explained in the second section. Filtering, visualizing and mining methods are applied for the case of Adana EMS system in the third section. Finally, the results are concluded in the last section of the paper.

\section{Materials and Methods}

\subsection{Adana EMS System}

Adana is one of the most populated cities in Turkey, located in the south of the country with an area of $14030 \mathrm{~km}^{2}$. The population is nearly 2.5 million people in the urban and rural area.

Adana EMS system has 33 stations in the center of the city and 14 stations located in rural areas as well mobilized stations including nearly 40 active ambulances. The call center receives in average 600 emergency calls per day. In 2016, the total number of calls received from all over the region was 187248. The cases are considered under two different types; emergency and transport calls.

The current ambulances and hospitals located in Adana is demonstrated in Figure 2. Due to the large scale of the city map, the locations are approximately chosen according to their destination points representing all the locations over the city.

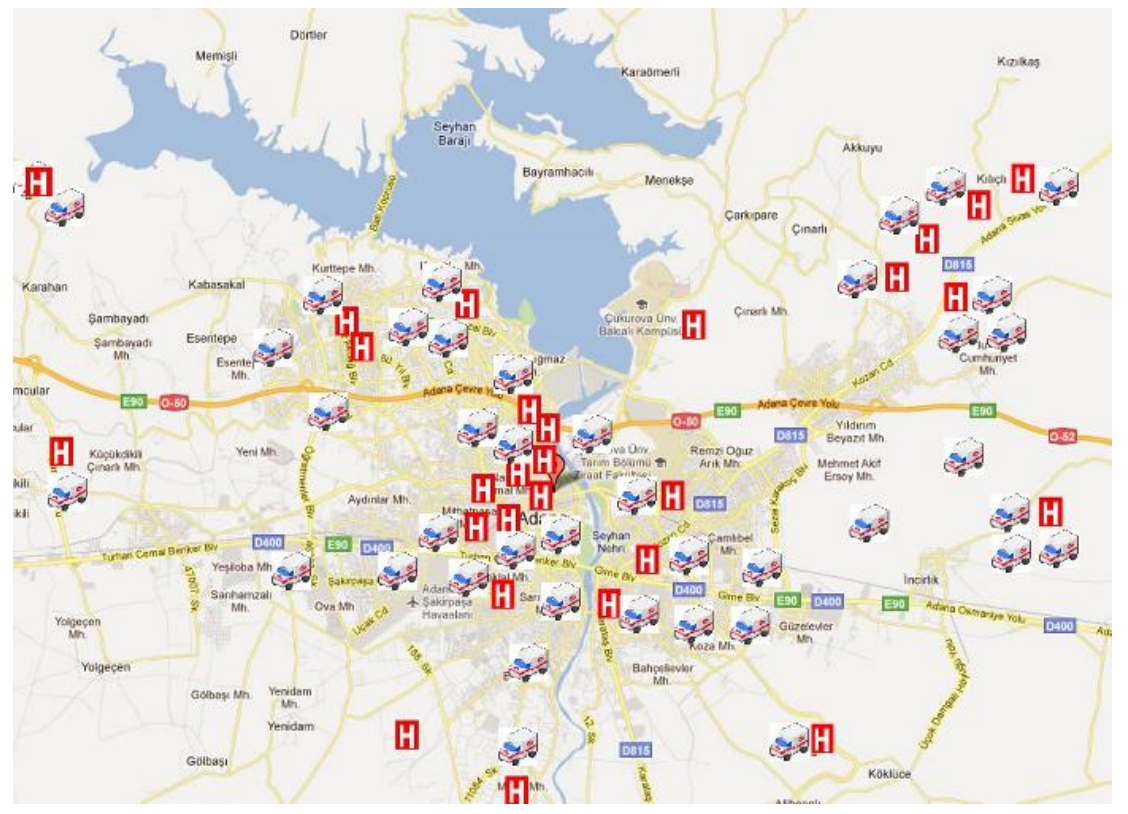

Figure 2. Location of ambulance stations and hospitals over the city of Adana

\subsection{Visualization of EMS System Data}

\subsubsection{EMS System Data Preprocessing}

EMS system data include 187248 cases. XESame is established by Buijs [18] and enables converting data to .xes file format to be compatible in process mining software, ProM. In order to implement ProM plug-ins, .xls file is edited and necessary arrangements are done before converting it to a .csv file. The .csv file is used in XESame for generating .xes file that is the compatible file format to be used in ProM. Furthermore, EMS system data is revised due to time zone problems. Thus, the date 
of the year is changed to 2012. However, we do not expect any lack of accuracy. A sample of event log is shown in Table 1.

Table 1. A sample of EMS system event logs

\begin{tabular}{|c|c|c|c|c|}
\hline Case ID & Event ID & & Properties & \\
\hline \multirow{7}{*}{ 2016-1 } & & Activity & Timestamp & Resource \\
\hline & 201611 & CallReceived & 01.01.2016 00:02:03 & ---- \\
\hline & 201612 & Prepareness & 01.01.2016 00:03:00 & ----- \\
\hline & 201613 & Move & 01.01.2016 00:04:00 & 7150 \\
\hline & 201614 & ArriveToScene & 01.01.2016 00:06:00 & 7150 \\
\hline & 201615 & Getcase & 01.01.2016 00:06:00 & 7150 \\
\hline & 201616 & DepartfromScene & 01.01.2016 00:33:00 & 7150 \\
\hline \multirow{5}{*}{$\begin{array}{l}2016-2 \\
2016-3\end{array}$} & 201621 & CallReceived & 01.01.2016 00:04:14 & \\
\hline & 201631 & CallReceived & $01.01 .201600: 12: 19$ & ----- \\
\hline & 201632 & Prepareness & 01.01.2016 00:13:00 & 7190 \\
\hline & 201633 & Move & 01.01.2016 00:14:00 & 7190 \\
\hline & 201634 & ArriveToHosp1 & 01.01.2016 00:20:00 & 7190 \\
\hline$\ldots \ldots$ & $\ldots \ldots \ldots+\cdots$ & $\ldots \ldots \ldots+\cdots \cdots$ & & $\cdots \cdots$ \\
\hline
\end{tabular}

\subsubsection{Filtering Event Logs}

187248 cases are recorded including 1667573 events before filtering. ProM enables the user to filter data from unwanted confusions and discover process model from event logs precisely. Due to the inconsistent and missing data, the EMS system event log is filtered by Simple Heuristic Filter plug-in. The traces are filtered as follows;

CallReceived > starting event

Prepareness \& TurnToStation > ending events

All other events are included in filtering phase. 149875 cases and 1332445 events remained after filtering phase. Almost 20 percent of data is observed unreliable or not recorded precisely. The number of minimum events per case and maximum events per case is given in Table 2. The number representing each activity is demonstrated in Table 3 .

Table 2. EMS system process event logs key data

\begin{tabular}{lc}
\hline Key Data & Quantity \\
\hline Process & 1 \\
Cases & 149875 \\
Events & 1332445 \\
Minimum number of events per case & 4 \\
Maximum number of events per case & 9 \\
\hline
\end{tabular}

Table 3. Activity names and their representative numbers

\begin{tabular}{lclc}
\hline Activity Name & Number & Activity Name & Number \\
\hline CallReceived & 1 & ArriveToHosp1 & 6 \\
Prepareness & 2 & ArriveToHosp2 & 7 \\
Move & 3 & DepartfromScene & 8 \\
ArriveToScene & 4 & FinalHospital & 9 \\
Getcase & 5 & DepartfFinalHospital & 10 \\
& & TurnToStation & 11 \\
\hline
\end{tabular}


Seven trace patterns put in order in respect of their percentage of occurrences over all the patterns are demonstrated in Table 4. The first trace representing 89 percent of cases has the pattern of CallReceived- Prepareness- Move-ArriveToScene- GetCase- DepartfromScene - FinalHosp DepartfFinalHospital - TurnToStation. This pattern illlustrates the emergency cases. The trace with the minimum number of events shows the pattern CallReceived-Prepareness- Move-TurnToStation representing the cases that are cancelled after ambulance is allocated to that case. These two patterns may occur if the patient is brought to the hospital by its relatives or the patient do not need any aid anyway or other miscellaneous reasons. The last trace is also similar to this case, howe ver the ambulance arrives the scene and then for some reason turns to station. The trace that is 11 percent of cases stands for transportation of patients to a final hospital while 0.17 percent of cases transportation to patients' home. The fourth trace representing 0.07 percent of cases point out that two of the hospitals does not accepted the patient, so the ambulance visits three hospitals for these cases. Finally, the fifth trace stands for the cases that the patient get well after first aid in the scene.

Table 4. EMS system traces after filtering and clearing inaccurate traces

\begin{tabular}{|c|c|c|}
\hline Trace Patterns & Occurrence & $\begin{array}{c}\text { Rate } \\
(\%)\end{array}$ \\
\hline 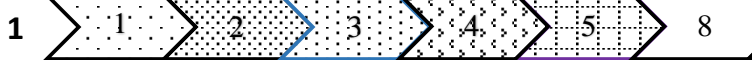 & 133874 & 89,32 \\
\hline 2 J $112 \mathrm{l}$ & 15631 & 10,4 \\
\hline$\sum 1$ & 251 & 0,17 \\
\hline $4 \mathrm{~d} / \mathrm{l}$ & 98 & 0,07 \\
\hline $5>1>$ & 19 & 0,01 \\
\hline $6 \sum 1 \%$ & 1 & 0,00 \\
\hline $7 \sum 1 \%$ & 1 & 0,00 \\
\hline
\end{tabular}

\subsubsection{EMS System Process Model}

Inductive Miner plug-in is used to obtain EMS system process model that is given in Figure 3. In the process model, the events flow in the direction of arrows and numbers in the boxes demonstrate the number of occurrence for the corresponding event. In the model, it is seen that while a few number of cases follow the transportation, a great number of cases follow the emergency flow as expected. The arrow above the ArriveToHosp 2 shows the number of cases that are executed from the preceding event but not executed from ArriveToHosp2. This model supports the patterns given in Table 4 . 


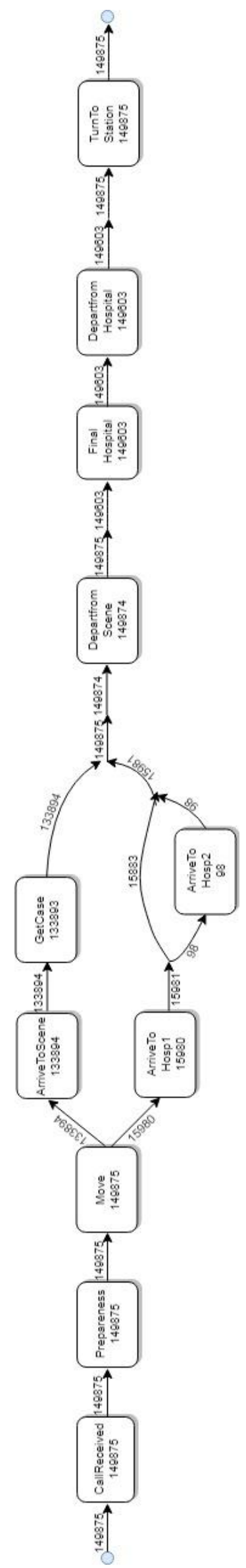

Figure 2. EMS system process model 
In the process model, the paths that have deviations are demonstrated in Figure 4. The numbers on the blue arrows show the cases that are executed from the corresponding event. The red dashed lines show the move-on model that represent the events in the event log but cannot be represented by the process model. A great amount of move-on model occurs for FinalHospital and DepartFinalHospital. It is obviously seen that EMS system process model in Figure 3 fits all the possible trace patterns in Table 4 while considering the move-on model in Figure 4.
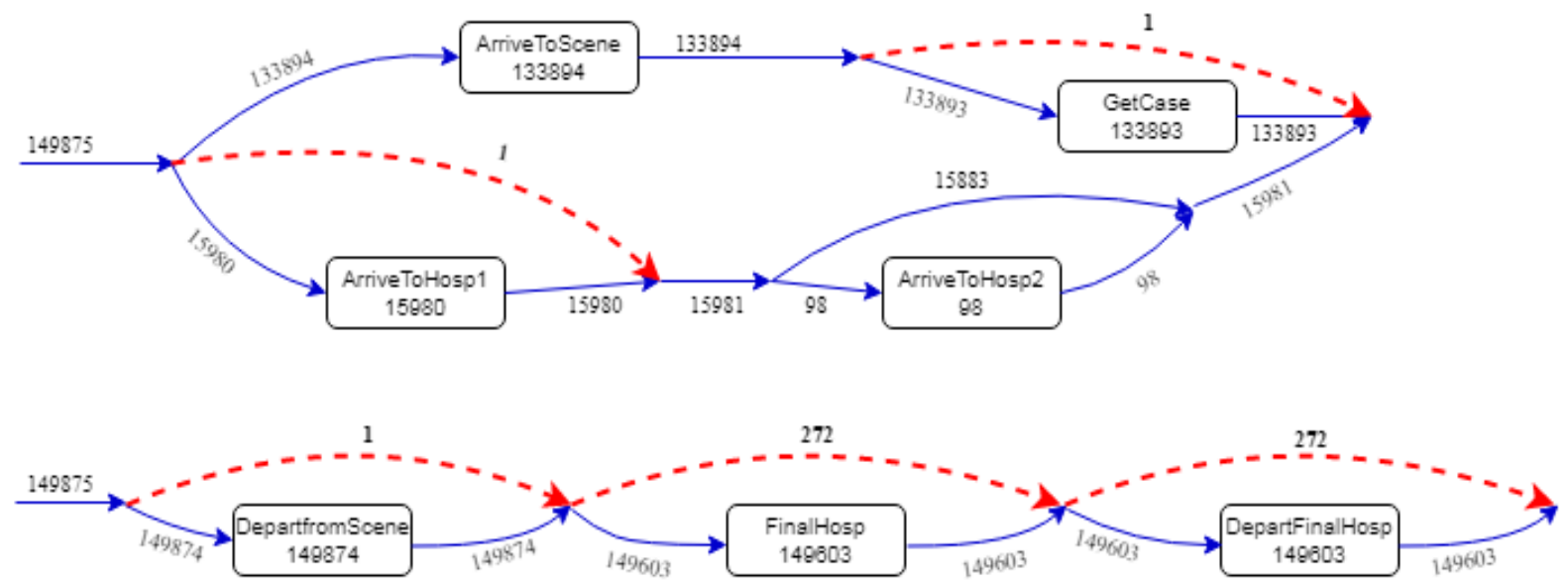

Figure 4. EMS system process model paths and deviations

\subsubsection{EMS System Fuzzy Model}

Another visualization of EMS system process given in Figure 5 is obtained by using Fuzzy Miner plug-in. In fuzzy model, thickness of the arrows shows the frequency of the corresponding event. The events ArriveToHosp1 and ArriveToHosp 2 stand for transportation and have less frequency than the emergency cases. The values inside the activity boxes show the occurrence rate of the corresponding event over all the cases. CallReceived and TurnToStation seem to be main events that exist in almost for all the cases. 


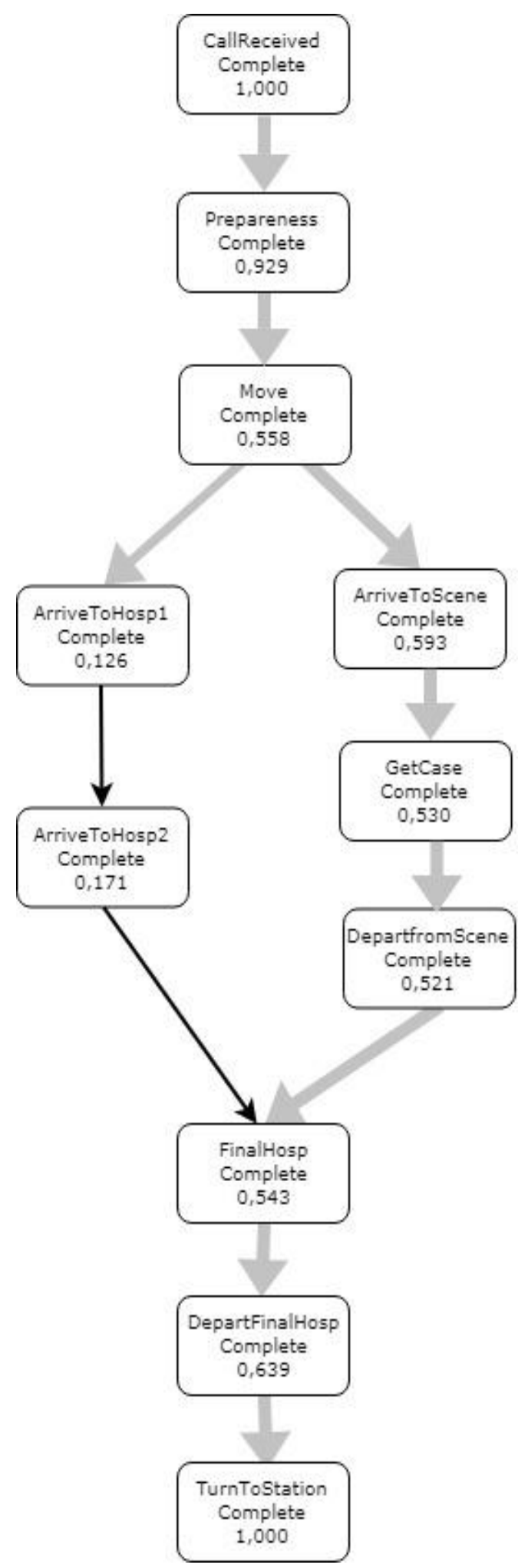

Figure 5. EMS system fuzzy model

\section{Discussion}

EMS system has many unreliable and inaccurate data due to the personnel who are responsible to record the cases and their timestamps. Despite of the fact that preprocessing face enables us to get more reliable and accurate data, it takes too much time to convert data in a compatible file format for process 
mining. Thus, the process mining methods are more convenient for the systems such as software, manufacturing in industry 4.0 , communication etc. where data are recorded automatically, not manually.

\section{Conclusion}

Discovering the real process model is necessary task to find the systems bottlenecks in a precise way and enables to enhance the system. Although the general flow of EMS system process is known, a process model that represents many types of different process flows is a good way of understanding the whole system in detail.

In this study, Adana EMS system data is used to demonstrate how to apply process mining techniques and tools while discovering process model and finding the variations in actual EMS system process based on the process model. The ProM Open software and the plug-ins which include process mining algorithms such as inductive miner, fuzzy miner, and heuristics for filtering are used in this application.

The results are useful to see the variations and the mostly or rarely occurring events and patterns. Two main patterns have the highest percentage of occurrences in seven different patterns. The emergency cases and the transportation between hospitals with 89 and 11 percent of total cases, respectively. Moreover, 7 percent of cases are important to be considered by EMS system managers. These cases transport patients from scene to a hospital, but based on the explanations of managers on this subject, the patient for some reason is not accepted by the first hospital and the ambulance must move to another hospital for these type of cases. It should be investigated whether these traces result in mortality because of the delay in transportation between hospitals.

For further studies, the reasons of unexpected trace patterns and deviations can be investigated in detail and the performance of the EMS system on timely basis may be analyzed, so the system performance can be enhanced.

\section{Acknowledgment}

This study is a part of research granted by The Scientific and Technical Research Council of Turkey (TUBITAK) International Post-Doctoral Research Fellowship Program in the framework of TUBITAK-BIDEB 2219. Thanks to Data Science Centre in Eindhoven University of Technology for their support and hospitality and also Adana Provincial Directorate of Health Center for providing data.

\section{References}

[1] Dick, W.F., (2003). Anglo-American vs. Franco-German emergency medical services system. Prehosp. Disaster Med., 18 (1), 29-35.

[2] Van der Aalst, W.M.P., Process mining: data science in action. Springer, Verlag Berlin Heidelberg, 2016.

[3] Van der Aalst, W.M.P., Weijters ,A.J.M.M., Maruster, L., (2004). Workflow mining: discovering process models from event logs. IEEE Transactions on Knowledge and Data Engineering, 16 (9), $1128-1142$.

[4] Weijters, A.J.M.M., Van der Aalst, W.M.P., De Medeiros, A.K.A., (2006).Process mining with the heuristics miner-algorithm. Technische Universiteit Eindhoven Technical Report WP , 166, 1-34. 
[5] De Medeiros, A.K.A., Weijters, A.J.M., Van der Aalst, W.P.M., (2005). Genetic process mining: a basic approach and its challenges. BPM 2005 International Workshops; Germany: Springer, 203215.

[6] Van der Aalst, W.M.P., Rubin ,V., Verbeek, H.M.W., Van Dongen, B.F., Kindler, E., Günther, C.W., (2010). Process mining: a two-step approach to balance between underfitting and overfitting. Software and Systems Modeling, 9(1), 87-111.

[7] Leemans, S.J.J., Fahland, D., Van der Aalst, W.M.P., (2013). Discovering block-structured process models from event logs: A constructive approach. In Applications and Theory of Petri Nets, Lecture Notes in Computer Science, Springer, Berlin, 7927, 311-329.

[8] Yurek, I., Birant, D., Birant, K.U., (2018). Interactive process miner: a new approach for process mining. Turkish Journal of Electrical Engineering \& Computer Sciences; 26, 1314-1328.

[9] Mans, R., Schonenberg, H., Leonardi, G., Panzarasa, S., Cavallini, A., Quaglini, S., Van der Aalst, W.M.P.,(2008). Process mining techniques: an application to stroke care. Studies in Health Technology and Informatics, 136, 573-578.

[10] Xiong, H.H., Zhou, M.C., Manikopoulos, C.N., (1994). Modeling and performance analysis of medical services systems using petri nets. In: IEEE Int. Conf. on Systems, Man and Cybernetic, .2339-2342.

[11] Rojas, E., Munoz-Gama, J., Sepulveda, M., Capurro, D., (2016). Process mining in healthcare: a literature review. Journal of Biomedical Informatics, 61, 224-236.

[12] Mans, R.S., Schonenberg, M.H., Song, M., Van der Aalst, W.M.P., Bakker, P.J.M.,(2008) Application of process mining in healthcare - a case study in a Dutch hospital. International Joint Conference on Biomedical Engineering Systems and Technologies, 425-438.

[13] Baker, K., Dunwoodie, E., Jones, R.G., Newsham, A., Johnson, O., Price, C.P., Wolstenholme, J., Leal, J., McGinley, P., Twelves, C., Hall, G., (2019). Process mining routinely collected electronic health records to define real-life clinical pathways during chemotherapy. International Journal of Medical Informatics, 103, 32-41.

[14] Alvarez, C., Rojas, E., Arias, M., Munoz-Gama, J., Sepulveda, M., Herskovic, V., Capurro, D., (2018). Discovering role interaction models in the emergency room using process mining. Journal of Biomedical Informatics, 78, 60-77.

[15] Yooa, S., Cho, M., Kima, E., Kima, S., Simb, Y., Yooc, D., Hwanga, H., Song, M., (2016). Assessment of hospital processes using a process mining technique: Outpatient process analysis at a tertiary hospital. International Journal of Medical Informatics, 88, 34-43.

[16] Yongzhong, C., Zhu, J., Guo, Y., Shi, C., (2018). Process mining-based medical program evolution. Computers and Electrical Engineering, 68, 204-214.

[17] Van der Aalst, W.M.P., (2012). Process mining: overview and opportunities. ACM Transactions on Management Information Systems, 3, 1-17.

[18] Buijs, J.C.A.M, Mapping Data Sources to XES in a Generic Way. Ph D. Thesis, Eindhoven University of Technology, Netherlands, 2010. 